NUMBER THEORY WEEK 2017

BANACH CENTER PUBLICATIONS, VOLUME 118

INSTITUTE OF MATHEMATICS

POLISH ACADEMY OF SCIENCES

WARSZAWA 2019

\title{
EXPLICIT AVERAGE ORDERS: NEWS AND PROBLEMS
}

\author{
OLIVIER RAMARÉ \\ CNRS/Institut de Mathématiques de Marseille, Aix Marseille Université, U.M.R. 7373 \\ Site Sud, Campus de Luminy, Case 907, 13288 Marseille Cedex 9, France \\ E-mail: olivier.ramare@univ-amu.fr
}

\begin{abstract}
We mix some of the novelties that have occurred recently in the field of explicit multiplicative number theory, together with some questions that have not been answered yet and with several new results.
\end{abstract}

1. Background. In the recent past several questions in multiplicative number theory have known some explicit extensions: instead of knowing that the problem is solvable provided the variables are large enough, a definite bound is given from which the result is true. Let us mention the representation of integers as sums of a prime (or a prime squared) and a square-free number by A. Dudek in [14] (and [15]), an explicit density estimate for Dirichlet $L$-series by the author in [36], the complete solutions of the odd Goldbach conjecture by H. Helfgott, a question that has been open for many decades, bounds on the number of Diophantine quintuples by T. Trudgian in [47] or M. Cipu in [7], and bounds on the least $k$-th power non-residue by E. Treviño in [46]. We cannot list all of them, but they have a particularity: computing the constants in the classical proof fails to do the job and new techniques are necessary. We focus in this article on a crucial auxiliary tool: averages of arithmetical functions. Rather than trying to define what arithmetical means here, let us say that the quantities to be averaged can roughly be sorted in three classes:

- Averages of the von Mangoldt $\Lambda$-function,

- Averages of non-negative multiplicative functions,

- Averages of oscillating multiplicative functions.

2010 Mathematics Subject Classification: Primary 11N37; Secondary 11A25, 11N56.

Key words and phrases: Multiplicative functions, explicit estimates, Möbius function.

The paper is in final form and no version of it will be published elsewhere. 
The first of these items have been extensively studied, while decent work exists concerning the second one. We consider here mainly the third item, and its implications on the second one. We will present work that bypasses the Perron formula, together with several problems that are still open, e.g., finding a decent lower bound for the absolute value of the Riemann zeta-function in a zero-free region.

Concerning primes, the situation is now essentially under control, thanks first to the pioneering work of J. B. Rosser in [41], extended together with L. Schoenfeld in [43]. Numerical verification of a partial Riemann hypothesis plays an important role and several authors intervened there. We only mention the most recent of them, namely D. Platt in [28. These computations concern also the zeros of $L$-functions, and this enables us to handle the distribution of primes in arithmetic progressions to some fixed modulus; the situation is less satisfactory there but getting tamed. Authors are now going towards derived quantities, e.g., $\sum \log (p) / p$ and Euler products, like O. Bordellès in [5], the present author in [30, the author's former student R. Mawia in [49] and P. Dusart in [16].

For non-negative multiplicative functions the convolution method usually works. Below we present two typical results in this area, that we use as starting points for three questions to quantify the progress and difficulties. For the first problem, we quote a result due to H. Cohen \& F. Dress in 1988, which is still unsurpassed.

Theorem 1.1 (H. Cohen \& F. Dress in [8]). For $D \geqslant 1004$, we have

$$
\sum_{d \leqslant D} \mu^{2}(d)=\frac{6 D}{\pi^{2}}+\mathcal{O}^{*}(0.1333 \sqrt{D}) .
$$

On assuming that $D \geqslant 438653$ (resp. $D \geqslant 82005$ ), we can replace 0.1333 by 0.02767 (resp. 0.036438).

We use our usual notation $f=\mathcal{O}^{*}(g)$ to mean that $|f| \leqslant g$. We produce in Corollary 3.4 an asymptotic error term that is asymptotically smaller, but only when $D \geqslant$ $10^{1468}$. Our method will have more impact on the second example we use for experiments. Here is a consequence of the above theorem.

Corollary 1.2. For $D>82005$, we have

$$
\sum_{d \leqslant D} \frac{\mu^{2}(d)}{d}=\frac{6}{\pi^{2}}\left(\log D+C_{2}\right)+\mathcal{O}^{*}\left(\frac{3 \times 0.036438}{\sqrt{D}}\right)
$$

with

$$
C_{2}=\gamma+2 \sum_{p \geqslant 2} \frac{\log p}{p^{2}-1}=1.71713765109062 \ldots
$$

When $D \geqslant 438653$, we can replace 0.036438 by 0.02767 . For $D \geqslant 1$, we can replace $3 \times 0.036438$ by 0.43 .

The proof of this corollary is straightforward and is delayed until Section 12 Theorem 3.2 and Corollary 3.3 below improve on the quality of this result as soon as $D \geqslant 10^{8}$ for the first one and as soon as $D \geqslant 3475$ for the second one. The third question we consider is linked with the Selberg sieve. Let us quote a result of J. Büthe. 
Theorem 1.3 (J. Büthe in [6]). For $D \geqslant 10^{9}$, we have

$$
\sum_{d \leqslant D} \frac{\mu^{2}(d)}{\varphi(d)}=\log D+c_{0}+\mathcal{O}^{*}\left(\frac{58}{\sqrt{D}}\right)
$$

where

$$
c_{0}=\gamma+\sum_{p \geqslant 2} \frac{\log p}{p(p-1)}=1.332582275733 \cdots
$$

This is after the work of H. Riesel and R. C. Vaughan in [39] and the present author in [31. The constant $c_{0}$ has been computed in [42, (2.10)]. Theorem 3.1 below improves considerably on this result.

Having these examples and results at hand, the main questions that remain are:

- Can we do better?

- What about oscillating multiplicative functions?

We will see that both questions are linked.

2. An analytical detour. When asking about possible improvements, it is interesting to see what the classical theory gives. The Dirichlet series associated with $\mu^{2}(n)$ is $\zeta(s) / \zeta(2 s)$ while the one associated with $\mu^{2}(n) / \varphi(n)$ reads

$$
\begin{aligned}
D(s) & =\prod_{p \geqslant 2}\left(1+\frac{1}{p^{s}(p-1)}\right) \\
& =\zeta(s+1) \frac{\zeta(4 s+4)}{\zeta(2 s+2)} \prod_{p \geqslant 2}\left(1-\frac{1}{p-1}\left(\frac{1}{p^{4 s+3}}+\frac{1}{p^{2 s+2}}-\frac{1}{p^{s+1}}-\frac{1}{p^{3 s+3}}\right)\right) .
\end{aligned}
$$

Proof. Let us give some tools on how to obtain such factorizations. We set $X=1 / p^{s+1}$ and $Y=p /(p-1)$, and then observe that

$$
(1+X Y)(1-X)\left(1+X^{2}\right)=1+(Y-1) X\left(1-X+X^{2}\right)-Y X^{4}
$$

from which the claimed formula follows on noticing that $1+X^{2}=\left(1-X^{4}\right) /\left(1-X^{2}\right)$.

We then use a truncated Perron formula, shift the line of integration to the left of the line $\Re s=-1 / 2$ but still within the zero-free region of $\zeta(2 s+2)$ and classically obtain

$$
\sum_{d \leqslant D} \mu^{2}(d) / \varphi(d)=\log D+C+\mathcal{O}\left(\exp (-c \sqrt{\log D}) D^{-1 / 2}\right)
$$

for some positive constant $c$. We cannot push the line of integration further to the left since we may otherwise encounter poles of $1 / \zeta(2 s+2)$ whose residues are uncontrolled. The situation for $\sum_{n} \mu^{2}(n)$ is exactly the same, since its Dirichlet series is $\zeta(s) / \zeta(2 s)$, except that the difficult line is now $\Re s=1$.

If one wants to compute the constants in the above proof, we encounter a major hurdle: bounds for $1 / \zeta(s)$ are hard to get. As a matter of fact, F. Dress asked this very question more than thirty years ago, though concerning $\sum_{n} \mu(n)$ rather than $\sum_{n} \mu(n)^{2}$, but the problem is the same. The best that has been obtained up to now has followed from the usual theory of the Riemann zeta-function. 
Theorem 2.1 (T. Trudgian in [4]). When $t \geqslant 45$ and $\sigma \geqslant 1-1 /(8 \log t)$,

$$
|1 / \zeta(s)| \leqslant 7 \cdot 10^{6} \log t
$$

Since the Riemann hypothesis has been checked for $|t| \leqslant 3 \cdot 10^{9}$, we can only ensure that $\log t \geqslant 21.8$ and the "constant" $7 \cdot 10^{6}$ behaves more like $t^{2 / 3}$. We use here the verification of D. Platt in [29] because it has been subject of a publication, but X. Gourdon has announced in 2004 in [21] a verification up to the height $2.445 \cdot 10^{12}$. This would sadly not make a large difference here.

3. Doing better. So, how to go beyond simple methods and remain explicit? We have changed "elementary" for "simple" in this sentence. Here "simple" means essentially that the constants do not accumulate to produce some useless error term.

We have recently managed to put a toe in this direction with P. Akhilesh in [38], and it is the history of this result that we recount below, with some comments and theorems in between. As it turns out, while writing this paper (in fact when preparing the talk), the author has improved on the result.

THEOREM 3.1. For $D>1$, we have

$$
\sum_{d \leqslant D} \frac{\mu^{2}(d)}{\varphi(d)}=\log D+c_{0}+\mathcal{O}^{*}\left(\frac{11}{\sqrt{D \log D}}\right),
$$

and equally for $D>1$, we have

$$
\sum_{d \leqslant D} \frac{\mu^{2}(d)}{\varphi(d)}=\log D+c_{0}+\mathcal{O}^{*}\left(\frac{61 / 25}{\sqrt{D}}\right) .
$$

The version with $\mathrm{P}$. Akhilesh has a 21 rather than 11 (and a 3.99 rather than $61 / 25=$ 2.44 ) but is valid for a larger family (with a coprimality condition $(d, q)=1$ added). The method here is simpler though it relies on the same mechanism we used. Note that Lemma 9.3 below has also an independent interest: it contains the result of some finite computation over the range $\left[1,10^{9}\right]$ that compares the error term with $1 / D^{3 / 4}$. We will first prove the following theorem.

ThEOREM 3.2. For $D \geqslant 1665$, we have

$$
\sum_{d \leqslant D} \frac{\mu^{2}(d)}{d}=\frac{6}{\pi^{2}}\left(\log D+C_{2}\right)+\mathcal{O}^{*}\left(\frac{7 / 20}{\sqrt{D \log D}}\right) .
$$

When $D>1$, it is enough to replace the $7 / 20$ with $3 / 5$ (and even by 0.56 ).

Again, Lemma 9.2 contains the result of some finite computation over the range $\left[1,10^{11}\right]$ that compares the error term with $1 / D^{3 / 4}$. With the help of Theorem 3.2 we can improve slightly on Corollary 1.2

Corollary 3.3. When $D \geqslant 3475$ is a real number, we have

$$
\sum_{d \leqslant D} \frac{\mu^{2}(d)}{d}=\frac{6}{\pi^{2}}\left(\log D+C_{2}\right)+\mathcal{O}^{*}\left(\frac{0.073}{\sqrt{D}}\right) .
$$

A simple summation by parts yields the following (together with some finite computations; all of these details are relegated to Section 10 . 
COROLlary 3.4. When $D>1$ is a real number, we have

$$
\sum_{d \leqslant D} \mu^{2}(d)=\frac{6}{\pi^{2}} D+\mathcal{O}^{*}\left(\frac{1.06 \sqrt{D}}{\sqrt{\log D}}\right) .
$$

This is better than the (basic) error term $\mathcal{O}^{*}(0.1333 \sqrt{D})$ of Theorem 1.1 only when $D \geqslant 10^{64}$ and better than $\mathcal{O}^{*}(0.02767 \sqrt{D})$ (from Theorem 1.1 when $D \geqslant 438653$ ) only when $D \geqslant 10^{1468}$.

Theorem 8.1 below is the first key to the results above. It will enable us to prove Theorem 3.2 from which we will deduce Theorem 3.1 by generalizing the method we devised with P. Akhilesh in [38. After explaining this one, we will set to tell how we came to Theorem 8.1 .

4. Setting the direction. We start our journey towards the proof of Theorem 3.1 and 3.2 by an Unbalanced Dirichlet Hyperbola Formula that we proved with P. Akhilesh in [38, Theorem 1.3]. See H. Montgomery [26, Lemma 1] for a similar idea.

THEOREM 4.1 (Unbalanced Dirichlet Hyperbola Formula). Let $(g(m))_{m \geqslant 1}$ be a sequence of complex numbers such that both series $\sum_{m \geqslant 1} g(m) / m$ and $\sum_{m \geqslant 1} g(m)(\log m) / m$ converge. We define $G^{\sharp}(x)=\sum_{m>x} g(m) / m$ and assume that $\int_{1}^{\infty}\left|G^{\sharp}(t)\right| d t / t$ converges. Let $A_{0} \geqslant 1$ be a real parameter. We have

$$
\sum_{n \leqslant D} \frac{(g \star \mathbb{1})(n)}{n}=\sum_{m \geqslant 1} \frac{g(m)}{m}\left(\log \frac{D}{m}+\gamma\right)+\int_{D / A_{0}}^{\infty} G^{\sharp}(t) \frac{d t}{t}+\mathcal{O}^{*}(\mathfrak{R})
$$

where $\mathfrak{R}$ is defined by

$$
\mathfrak{R}=\left|\sum_{1 \leqslant a \leqslant A_{0}} \frac{1}{a} G^{\sharp}\left(\frac{D}{a}\right)+G^{\sharp}\left(\frac{D}{A_{0}}\right)\left(\log \frac{A_{0}}{\left[A_{0}\right]}-R\left(\left[A_{0}\right]\right)\right)\right|+\frac{6 / 11}{D} \sum_{m \leqslant D / A_{0}}|g(m)|
$$

where $\left[A_{0}\right]$ is the integer part of $A_{0}$, while the remainder $R(X)=\sum_{n \leqslant X} 1 / n-\log X-\gamma$.

The constant $6 / 11=0.545 \cdots$ is commented on in [38, Lemma 2.1] and can be replaced by the optimal $2(\log 2+\gamma-1)=0.540 \cdots$. This theorem tells us that the key to improving the estimate for $\mu^{2}(d) / d$ is to get some non-trivial bound for $\sum_{\ell>y} \mu(\ell) / \ell^{2}$. In the same manner, the key to $\mu^{2}(d) / \varphi(d)$ is to estimate non-trivially

$$
\sum_{\substack{\ell^{2} k>y,(\ell, k)=1}} \frac{\mu^{2}(k) \mu(\ell)}{k \varphi(k) \ell \varphi(\ell)}
$$

Here, by "non-trivially", we mean exploiting the cancellation offered by the $\mu(\ell)$ factor. This is the path we followed in [38] but we shall see in Section 8 that another path is possible.

The above theorem covers the case of a convolution product $g \star \mathbb{1}$, but it may be useful to have a more general case. Let $h$ be an arithmetical function (i.e. simply a sequence of complex numbers $\left.(h(n))_{n \geqslant 1}\right)$ and $K_{0}$ and $K_{1}$ be two complex numbers. We define the remainder $R_{h}$, which in fact depends also on $K_{0}$ and $K_{1}$, by

$$
\forall X \geqslant 1, \quad \sum_{n \leqslant X} h(n) / n=K_{0}\left(\log X+K_{1}\right)+R_{h}(X) .
$$


Theorem 4.2 (Generalized Unbalanced Hyperbola Formula). Let $(g(m))_{m \geqslant 1}$ be a sequence of complex numbers such that both series $\sum_{m \geqslant 1} g(m) / m$ and $\sum_{m \geqslant 1} g(m)(\log m) / m$ converge. We define $G^{\sharp}(x)=\sum_{m>x} g(m) / m$ and assume that $\int_{1}^{\infty}\left|G^{\sharp}(t)\right| d t / t$ converges. Let $A_{0} \geqslant 1$ be a real parameter and $h, K_{0}, K_{1}$ and $R_{h}$ be as above. We have

$$
\sum_{n \leqslant D} \frac{(g \star h)(n)}{n}=K_{0} \sum_{m \geqslant 1} \frac{g(m)}{m}\left(\log \frac{D}{m}+K_{1}\right)+K_{0} \int_{D / A_{0}}^{\infty} G^{\sharp}(t) \frac{d t}{t}+\mathcal{O}^{*}\left(\Re_{h}\right)
$$

where $\mathfrak{R}_{h}$ is defined by

$$
\begin{aligned}
\mathfrak{R}_{h}= & \left|\sum_{a \leqslant A_{0}} \frac{h(a)}{a} G^{\sharp}\left(\frac{D}{a}\right)+G^{\sharp}\left(\frac{D}{A_{0}}\right)\left(K_{0} \log \frac{A_{0}}{\left[A_{0}\right]}-R_{h}\left(\left[A_{0}\right]\right)\right)\right| \\
& +\sum_{m \leqslant D / A_{0}}\left|\frac{g(m)}{m} R_{h}\left(\frac{D}{m}\right)\right|
\end{aligned}
$$

where $\left[A_{0}\right]$ is the integer part of $A_{0}$.

Partial proof of Theorem 4.2. We start by proving the following formula:

$$
\begin{aligned}
\sum_{n \leqslant D} \frac{(g \star h)(n)}{n}= & K_{0} \sum_{m \geqslant 1} \frac{g(m)}{m}\left(\log \frac{D}{m}+K_{1}\right)+K_{0} \int_{D}^{\infty} G^{\sharp}(t) \frac{d t}{t} \\
& -K_{0} K_{1} G^{\sharp}(D)+\sum_{m \leqslant D} \frac{g(m)}{m} R_{h}\left(\frac{D}{m}\right) .
\end{aligned}
$$

This identity is linear in $g$, so it is enough to prove it for any positive integer $k$ for $g=\delta_{:=k}$, i.e. the function that takes the value 1 at $k$ and the value 0 everywhere else. In this case, $G^{\sharp}(t)$ is 0 when $t>k$ and equal to $1 / k$ otherwise, while $(g \star h)(n)$ is $h(n / k)$ when $k$ divides $n$ and 0 otherwise. The identity to prove reduces to

$$
\sum_{m \leqslant D / k} \frac{h(m)}{k m}=K_{0} \frac{1}{k}\left(\log \frac{D}{k}+K_{1}\right)+\frac{K_{0}}{k} \delta_{D<k} \log \frac{k}{D}-\delta_{D<k} \frac{K_{0} K_{1}}{k}+\frac{\delta_{D \geqslant k}}{k} R_{h}(D / k) .
$$

On splitting according to whether $D<k$ or not, the reader will readily check the identity. In order to continue, we need to rewrite the part

$$
\sum_{D / A_{0}<m \leqslant D} \frac{g(m)}{m} R_{h}\left(\frac{D}{m}\right) .
$$

This is done in rather technical Covering Remainder Lemma 11.1 that we prove in Section 11

As one can see, the preliminary step to Theorem 3.2 is to find a manner to handle the Möbius function. The analytical way seems to be ruled out. On the other side and as we have seen, lots of work has been done for the von Mangoldt $\Lambda$-function, and it would be great to inherit from it.

5. Leading theme. The Dirichlet series associated with the von Mangoldt $\Lambda$-function is $-\zeta^{\prime}(s) / \zeta(s)$. The Euler product relates to primes, but by looking at it as $-\zeta^{\prime}(s)$, defined in terms of integers, divided by $\zeta$, equally defined in terms of integers, we have a way to relate primes to integers. This is in short the Riemann program. But for this program to 
work, we need $\zeta(s)$ not to vanish and this is the crucial point. The factor $\zeta^{\prime}(s)$ is most probably superfluous. Pursuing this line of thought, we see that the distribution of the primes should be "equivalent" to the distribution of the Dirichlet coefficients of $1 / \zeta(s)$, i.e. to $(\mu(n))_{n \geqslant 1}$. This philosophy motivates the following theorem.

THEOREM 5.1 (Axer-Landau Equivalence Theorem [23, [1 \& 24]). The five propositions are equivalent:

(1) \#\{primes $\leqslant x\}$ is asymptotic to $x / \log x$.

(2) $\psi(x)=\sum_{n \leqslant x} \Lambda(n)$ is o(x).

(3) $M(x)=\sum_{d \leqslant x} \mu(d)$ is $o(x)$.

(4) $m(x)=\sum_{d \leqslant x} \mu(d) / d$ is $o(1)$.

(5) $\check{m}(x)=\sum_{d \leqslant x} \mu(d) \log (x / d) / d$ is $1+o(1)$.

A quantitative version of this theorem would be numerically efficient! The equivalence between (1) and (2) is trivial and we have given some ground for the equivalence of (2) and (3). The statement that (2) implies (4) is somewhat surprising and comes from E. Landau's thesis in [23], while that the statement (3) implies (4) is due to A. Axer in [1]. We shall tell more on this subject later and it is in fact the subject of the survey [37]. The statement (5) concerning the function $\check{m}$ is a surprising addition.

In between, let us consider a related question.

Question 1. Can one bound efficiently $|1 / \zeta(s)|$, in some zero-free region, in terms of $\psi(x)$ ?

In connection with this question we propose how to go from $\psi(x)$ to $M(x)$ in a not too bad manner.

6. From the primes to the Möbius function. Let us continue our journey around the Axer-Landau Equivalence Theorem. We first notice that W.-B. Zhang has exhibited in [50] a Beurling system of integers where one has $M_{\mathcal{P}}(x)=o(x)$ without $\psi_{\mathcal{P}}(x) \sim x$. Our final destination being numerical estimates, we are however more interested in the reverse implication, i.e. to derive bounds for $M$ from bounds for the primes. This phenomenon is detailed in the survey [37]. This problem has been studied by A. Kienast in [22] and by L. Schoenfeld in [44, and they proceeded as the author later did in [33] by using some combinatorial identities. Tough we indeed get results, the process we use is heavy and saves only some power of log. The higher this power, the more difficult the proof becomes; but we have been unable to find a path that would enable a power saving. The family of identities the author produced is simply more efficient than the one used by A. Kienast. It is better to refer the reader to the cited paper and to only give here the general flavour. The first interesting case reads

$$
\sum_{\ell \leqslant x} \mu(\ell) \log ^{2} \ell=\sum_{d \ell \leqslant x} \mu(\ell)(\Lambda \star \Lambda(d)-\Lambda(d) \log d) .
$$

It is worth mentioning that the Selberg identity that is used for proving elementarily the Prime Number Theorem is $\Lambda \star \Lambda(d)+\Lambda(d) \log d=\left(\mu \star \log ^{2}\right)(d)$ and that, assuming this theorem, both factors $\Lambda \star \Lambda(d)$ and $\Lambda(d) \log d$ contribute equally to the average. In particular, the function $\Lambda \star \Lambda(d)-\Lambda(d) \log d$ should be looked upon as a remainder term. 
We get information of its average order by using the Dirichlet hyperbola formula; it would most probably be better to use an explicit expression in terms of the zeros directly, but this involves the residues of $\left(\zeta^{\prime} / \zeta\right)^{2}$ and there lacks a control of those, while the residues of $\zeta^{\prime} / \zeta$ are well understood. Some more thought discloses that we need essentially the $L^{1}$-norm of such residues. Since they are non-negative integers for $\zeta^{\prime} / \zeta$, we may as well compute their simple average, which is readily achieved by a contour integration that has most of its path outside the critical strip. No such phenomenon is known to occur for $\left(\zeta^{\prime} / \zeta\right)^{2}$.

The reader may be wary of the Möbius factor that appears on the right-hand side of (3), but only one such factor appears. It is maybe more apparent in the next identity of this series:

$$
\sum_{\ell \leqslant x} \mu(\ell) \log ^{3} \ell=\sum_{d \ell \leqslant x} \mu(\ell)\left(\Lambda \star \Lambda \star \Lambda(d)-3 \Lambda \star(\Lambda \log )(d)+\Lambda(d) \log ^{2} d\right) .
$$

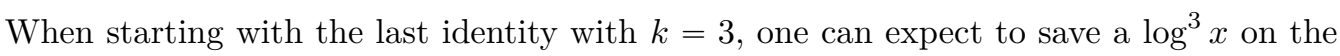
trivial estimate $x$, but the presence of the Möbius factor on the right-hand side reduces that to a saving of one $\log x$ less, so $\log ^{2} x$. This is because the Dirichlet hyperbola method is not used, though one may employ a recursion process: indeed, L. Schoenfeld does that, followed by H. Cohen, F. Dress \& M. El Marraki in [9], 13] and [17]. The present author did not introduce such a step as it is numerically costly, but a more careful treatment is possible here.

TheOREM 6.1 ([33]). When $D \geqslant 464402$, we have

$$
\left|\sum_{d \leqslant D} \mu(d)\right| / D \leqslant \frac{0.0146 \log D-0.1098}{(\log D)^{2}} .
$$

QUeSTION 2. Can one introduce a recursion step in the proof of the above result to increase the explicit saving?

The 0.0146 is to be compared with $3 / 28$ from [17]. And, yes, it is amazing that one is not able to do much better, like $\leqslant 10^{-6} D$ for sensible values of $D$ (our result requires $D \geqslant 10^{6500}$ to reach such a conclusion) as in the case for the primes (see [19]).

The present author believes (as already stated elsewhere) that there exists $A>1$ such that

$$
|M(x)| \stackrel{?}{\ll} \max _{x / A<y \leqslant x A}|\psi(y)-y| / y+x^{-1 / 4} .
$$

Question 3. Can one bound $1 / \zeta(s)$ in terms of $M(x)$ in some zero-free region?

This question is trickier than it looks and the classical expression

$$
\frac{1}{\zeta(s)}=s \int_{1}^{\infty} M(x) \frac{d x}{x^{s+1}}
$$

is apparently not sufficient to go beyond $\Re s=1$.

7. From $M(x)$ to $m(x)=\sum_{d \leqslant x} \mu(d) / d$. The reader may believe than an integration by parts does the job, but there is a catch. Indeed, on the formula

$$
m(x)=\frac{M(x)}{x}+\int_{1}^{x} \frac{M(t) d t}{t^{2}},
$$


we see that the assumption $M(x)=o(x)$ is not enough to ensure that $m(x)$ is even bounded! The same problem occurs when going from the Chebyshev $\psi$-function to $\tilde{\psi}(x)=$ $\sum_{n \leqslant x} \Lambda(n) / n$. In this case, H. Diamond \& W.-B. Zhang in [12] found a system of Beurling integers where $\psi(x)$ is equivalent to $x$, but $\tilde{\psi}(x)-\log x$ is unbounded. No such counterexample has been found for the Möbius function, though the present author expects a similar phenomenon to occur.

We thus have to use some special property of the actual sequence we consider. Recall that we are seeking quantitative results. In the case of the primes, it took the author quite a while, but D. Platt and the present author finally cleared the situation in [32] and [30]. Concerning the Möbius function, A. Axer in [1] produced a qualitative answer. M. El Marraki in a preprint [18, that has only known a very confidential dissemination, used an identity to do so, and we followed the same path. Our new ingredient is an identity due to M. Balazard in [4]. Here is our result.

TheOREM 7.1 ([35]). When $D \geqslant 463421$, we have

$$
\left|\sum_{d \leqslant D} \mu(d) / d\right| \leqslant \frac{0.0144 \log D-0.1}{(\log D)^{2}}
$$

When $D \geqslant 97000$, we have

$$
\left|\sum_{d \leqslant D} \mu(d) / d\right| \leqslant \frac{1}{69 \log D}
$$

M. El Marraki in the aforementioned preprint had 4.5 instead of 69 . Since the magical tool is a collection of identities, we recall one of these identities, so that the reader can imagine the beasts we are looking at. In the proof of Proposition 6 of [3], M. Balazard produces the following identity, valid for $x \geqslant 1$ :

$$
m(x)=\frac{M(x)}{x}+\frac{4\left(1-x^{-1}\right)^{2}}{x}-\frac{4\left(1-x^{-1}\right)^{3}}{3 x^{2}}+\frac{1}{x} \int_{1}^{x} M(x / t) \varepsilon_{1}^{\prime}(t) d t
$$

where

$$
\varepsilon_{1}^{\prime}(t)=\left(\frac{(2\{t\}-1) t+\{t\}-\{t\}^{2}}{t^{2}}\right)^{2} .
$$

From that he deduces the following theorem.

Theorem 7.2 (M. Balazard in [4]). When $D \geqslant 1$, we have

$$
|m(D)| \leqslant \frac{|M(D)|}{D}+\frac{1}{D^{2}} \int_{1}^{D}|M(t)| d t+\frac{8}{3 D} .
$$

It turns out that M. Balazard was studying and trying to put some order in a collection of identities produced by R. A. MacLeod in [25]. This very paper is entitled A curious identity for the Möbius function, and indeed, the question is to know whether such identities are ad hoc historical curiosities or whether some more information is lying there.

The situation has been further cleared by a former student of mine, F. Daval [10], in the following theorem. 
Theorem 7.3 (F. Daval in [10]). Select $h:[0,1] \rightarrow \mathbb{C}$, continuous and such that $\int_{0}^{1} h(u) d u=1$. When $x \geqslant 1$, we have

$$
m(x)-\frac{M(x)}{x}-\frac{1}{x} \int_{1 / x}^{1} \frac{h(y)}{y} d y=\frac{1}{x} \int_{1}^{x} M(x / t)\left(1-\frac{1}{t} \sum_{n \leqslant t} h(n / t)\right) d t .
$$

So we can handle the convolution of $M$ against any "Riemann integral-remainder"! On selecting $h=1$, one recovers the classical Meissel identity, while the choice $h=2 t$ leads to the first MacLeod identity.

The class of these "Riemann integral-remainder" is not yet clear. Given $f$ over $[0,1]$, say continuous, with integral equal to one, can it be approximated by such a remainder term, or more precisely, what is the class of functions attained?

We show in Theorem 7.4 below that we get every identity in this manner, at least when $h$ is assumed to be $C^{1}$.

7.1. The problem at large. Let us try to formalize the problem. We start from a regular function $F:[1, \infty) \rightarrow \mathbb{C}$, for instance $F(t)=1$ or $F(t)=\log t$. The question is to find two functions $H$ and $G$ and a constant $C$ such that, for any $x \geqslant 1$, we have

$$
\sum_{n \leqslant x} \frac{\mu(n)}{n} F(x / n)-C \frac{M(x)}{x}=\frac{1}{x} \int_{1}^{x} M(x / t) G(t) d t+H(x) .
$$

We assume that $H$ is smooth and "small". To avoid solutions that would result from integration by parts, we assume that

$$
\int_{1}^{\infty}|F(t)| d t / t=\infty, \quad \int_{1}^{\infty}|G(t)| d t / t<\infty .
$$

Indeed, an integration by parts yields the formula

$$
\frac{1}{x} \int_{1}^{x} M(x / t) G(t) d t=\sum_{n \leqslant x} \frac{\mu(n)}{n} F(x / n)
$$

but we have

$$
\infty=\int_{1}^{\infty}|F(t)| \frac{d t}{t}=\int_{1}^{\infty}\left|\frac{1}{t} \int_{1}^{t} G(y) d y\right| \frac{d t}{t} \leqslant \int_{1}^{\infty}|G(y)| d t / t<\infty,
$$

which contradicts our conditions.

Equality (5) looks like a functional transform from $F$ to $G$, but there is a lot of slack! Indeed, when $F=1$ or when $F(t)=\log t$, the vector space of solutions is infinite dimensional.

Note that, by looking at what happens at $x=1$, we find that $C=F(1)-H(1)$.

\subsection{A completeness result when $F=1$}

THEOREM 7.4. Assume relation (5) holds with $F=1, G$ and $H$ being $C^{2}$ over $[1, \infty)$. Then $C=1$ and the function defined over $[0,1]$ by $h(z)=(H(1 / z) / z)^{\prime} / z$ is $C^{1}$ over $[0,1]$, satisfies $\int_{0}^{1} h(z) d z=1$ and we have, when $x \geqslant 1$,

$$
G(x)=1-\frac{1}{x} \sum_{n \leqslant x} h(n / x)
$$


We did not try to reach minimal hypotheses and, in particular, to relax the regularity assumption on $G$.

Proof. When $\Re s<0$, we find that

$$
\int_{1}^{\infty} \sum_{n \leqslant x} \frac{\mu(n)}{n} x^{s-1} d x=\sum_{n \geqslant 1} \frac{\mu(n)}{n} \int_{n}^{\infty} x^{s-1} d x=\frac{-1}{s \zeta(1-s)} .
$$

Note also that $(s-1) \int_{1}^{\infty} M(x) x^{s-2} d x=1 / \zeta(1-s)$. Again when $\Re s<0$, we find that

$$
\begin{aligned}
\int_{1}^{\infty} \frac{1}{x} \int_{1}^{x} M(x / t) G(t) d t x^{s-1} d x & =\int_{1}^{\infty} \frac{1}{x} \sum_{n \leqslant x} \mu(n) \int_{1}^{x / n} G(t) d t x^{s-1} d x \\
& =\sum_{n \geqslant 1} \mu(n) \int_{1}^{\infty} \int_{n t}^{\infty}(x / n)^{s-1} n^{s-1} \frac{d x}{x} G(t) d t \\
& =\sum_{n \geqslant 1} \mu(n) \int_{1}^{\infty} \int_{t}^{\infty} x^{s-1} n^{s-1} \frac{d x}{x} G(t) d t \\
& =\sum_{n \geqslant 1} \mu(n) n^{s-1}\left(\frac{-1}{s-1}\right) \check{G}(s)
\end{aligned}
$$

on denoting the Mellin transform of $G$ by $\check{G}(s)$, i.e.

$$
\check{G}(s)=\int_{1}^{\infty} G(x) x^{s-1} d x .
$$

This means, with respect to the usual theory where the functions are defined over $[0, \infty)$, that we extend $G$ to this interval by setting $G(x)=0$ when $x \in[0,1)$. We infer from these computations that, when $\Re s<0$,

$$
\check{G}(s)=\frac{s-1}{s}-C+\zeta(1-s)(s-1) \check{H}(s)
$$

or also

$$
\check{G}(s)=1-C+\frac{1}{s}(-(s-1) \check{H}(s)-1)+\left(\zeta(1-s)+\frac{1}{s}\right)(s-1) \check{H}(s) .
$$

Since we ask that $\check{G}(0)$ exists, we need $\check{H}(0)=1$. Furthermore, (5) with $x=1$ gives us $H(1)=1+C$. And since $H$ is $C^{2}$, its Mellin transform decreases as fast as $1 /|s|^{2}$ when $s$ is large. In particular we should account for the $1-C-s^{-1}$ of $\check{G}$. The 1 is the Mellin transform of the Dirac measure at $x=1$ and $-1 / s$ of the function $\mathbb{1}_{[1, \infty)}$, but also of the $Y$ function defined by

$$
Y(x)= \begin{cases}0 & \text { when } x \in[0,1), \\ \frac{1}{2} & \text { when } x=1 \\ 1 & \text { when } x>1\end{cases}
$$

This function is better suited for inverse Mellin transform. Since $G$ is regular, its Mellin transform has no Dirac part, which means that $1-C=0$ and thus

$$
\check{G}(s)=\check{Y}(s)+\zeta(1-s)(s-1) \check{H}(s) .
$$


Let us start toward a reciprocal statement. We first notice that

$$
(s-1) \check{H}(s)=\left[x H(x) x^{s-1}\right]_{1}^{\infty}-\int_{1}^{\infty}(x H(x))^{\prime} x^{s-1} d x .
$$

We set

$$
h(1 / y) / y=(y H(y))^{\prime}, \quad H(x)=\frac{1}{x} \int_{1}^{x} h(1 / y) d y / y .
$$

The equation $\check{H}(0)=1$ translates into

$$
\int_{1}^{\infty} \int_{1}^{x} h(1 / v) \frac{d v}{v} \frac{d x}{x^{2}}=\int_{1}^{\infty} \int_{1 / x}^{1} h(u) \frac{d u}{u} \frac{d x}{x^{2}}=\int_{0}^{1} h(u) d u=1 .
$$

We infer from this by inverse Mellin transform that

$$
\begin{aligned}
G(x)-Y(x) & =\frac{1}{2 i \pi} \int_{-\frac{1}{2}-i \infty}^{-\frac{1}{2}+i \infty} \zeta(1-s)(s-1) \check{H}(s) x^{-s} d s \\
& =\sum_{n \geqslant 1} \frac{1}{n} \frac{1}{2 i \pi} \int_{-\frac{1}{2}-i \infty}^{-\frac{1}{2}+i \infty}(s-1) \check{H}(s)(x / n)^{-s} d s=-\frac{1}{x} \sum_{n \leqslant x} h(n / x)
\end{aligned}
$$

recovering F. Daval's identity.

7.3. A functional approach when $F=1$. Starting from Theorem 7.3 and, remembering the identities of MacLeod in Balazard's form, we aim at writing the integral with $M$ in the form $\int M(x / t) f^{\prime}(t) d t$. With this goal in sight we note that

$$
\int_{0}^{x}\left(1-\frac{1}{t} \sum_{n \leqslant t} h(n / t)\right) d t=\int_{0}^{1}\{u x\} \frac{h(u)}{u} d u .
$$

So, given $f:[1, \infty) \rightarrow \mathbb{C}$, we would like to solve $f(x)=\int_{0}^{1}\{u x\} \frac{h(u)}{u} d u$. The change of variable $y=1 / x$ leads to the problem: given $g:[0,1] \rightarrow \mathbb{C}$, solve $g(y)=\int_{0}^{1} \frac{\{u / y\}}{u / y} h(u) d u$. The operator, say $T$, over the Hilbert space $L^{2}([0,1])$ which associates $\int_{0}^{1} \frac{\{u / y\}}{u / y} h(u) d u$ to $h$ is a Hilbert-Schmidt, compact and contracting operator. Indeed, we readily check that the kernel $(u, y) \mapsto \frac{\{u / y\}}{u / y}$ belongs to $L^{2}\left([0,1]^{2}\right)$ and then, we for instance use the classical textbook [20] (around equations (9.6)-(9.8)). Since

$$
\begin{aligned}
\int_{0}^{1} \int_{0}^{1}\left|\frac{\{u / y\}}{u / y}\right|^{2} d u d y & =\int_{0}^{1} \int_{0}^{1 / y}\left|\frac{\{z\}}{z}\right|^{2} d z y d y \\
& \leqslant \int_{0}^{1}\left(1+\int_{1}^{\infty} \frac{\{z\}}{z^{2}} d z\right) y d y=\frac{1}{2}(2-\gamma)<1
\end{aligned}
$$

we see by invoking the Cauchy-Schwarz inequality that $T$ is strictly contracting. Indeed, let $\varphi$ be a normalized eigenvector of $T$ associated with the eigenvalue $\lambda$, we have

$$
\begin{aligned}
|\lambda|^{2} & =\int_{0}^{1}|\lambda \varphi(y)|^{2} d y=\int_{0}^{1}\left|\int_{0}^{1} \frac{\{u / y\}}{u / y} \varphi(u) d u\right|^{2} d y \\
& \leqslant \int_{0}^{1} \int_{0}^{1}\left|\frac{\{u / y\}}{u / y}\right|^{2} d u \int_{0}^{1}|\varphi(u)|^{2} d u d y \leqslant \frac{1}{2}(2-\gamma) .
\end{aligned}
$$

The general theory tells us that there exists a sequence of complex numbers $\left(\lambda_{n}\right)_{n}$ tending to zero (arranged in non-increasing order of their absolute value), and two orthonormal 
sequences of functions $\left(\psi_{n}\right)_{n}$ and $\left(\varphi_{n}\right)_{n}$ such that

$$
\int_{0}^{1}\{u / y\} \frac{h(u)}{u} d u=\sum_{n \geqslant 1} \lambda_{n} \int_{0}^{1} h(u) \overline{\psi_{n}(u)} d u \varphi_{n}(y)
$$

for every $y \in[0,1]$. By the paper [45] of D. W. Swann, this operator is of Shatten class $p$ for every $p>1$ (meaning that $\sum_{n}\left|\lambda_{n}\right|^{p}<\infty$ ), and the author suspects it is not of trace class. The above decomposition is a consequence of the general theory of integral operators and a more specific study should be able to disclose arithmetical properties.

The description of the operator $T$ is possibly linked with the Nyman-Beurling criteria. Indeed, this asserts that the characteristic function of $(0,1]$ belongs to the closure in $L^{2}(0, \infty)$ of the set of functions defined by $y \mapsto\{u / y\}$ for any parameter $u \in(0,1)$ (see for instance [2]). The function $g(y) / y$ would belong to this closure if it were defined for $y \in(0, \infty)$ and not only for $y \in(0,1]$. We close this aside and resume the main course.

Question 4. Can one describe explicitly the triples $\left(\lambda_{n}, \varphi_{n}, \psi_{n}\right)$ ?

7.4. Other streams of identities. Continuing our exploration of this kind of identities, we are led to the choice $F(x)=(\log x)^{k}$ in (5), for non-negative integer $k$. J.-P. Gram had already an identity of this kind in 1884! R. A. MacLeod and M. Balazard produced a full bunch of other identities, but a nice theory like the one of F. Daval in Theorem 7.3 is still missing.

Rather than expanding on this subject, the author prefers to concentrate here on one application. Here is an identity proved in [35] by following [4] and [3]. For every $x \geqslant 1$, we have

$$
\begin{aligned}
\sum_{n \leqslant x} \frac{\mu(n)}{n} \log \left(\frac{x}{n}\right)-1= & \frac{6-8 \gamma}{3 x}-\frac{5-4 \gamma}{x^{2}}+\frac{6-4 \gamma}{3 x^{4}} \\
& +\left(\frac{1}{2}-\gamma\right) \frac{M(x)}{x}-\frac{1}{x} \int_{1}^{x} M(x / t) h^{\prime}(t) d t
\end{aligned}
$$

where $h$ is a function that satisfies $t^{2}\left|h^{\prime}(t)\right| \leqslant \frac{7}{4}-\gamma$. This function can be very explicitly described. This leads to the following unexpected result.

TheOREM 7.5 ([35, Theorem 1.5]). When $D \geqslant 3861$, we have

$$
\left|\sum_{d \leqslant D} \frac{\mu(d) \log (D / d)}{d}-1\right| \leqslant \frac{0.00252 \log D-0.0077}{(\log D)^{2}} .
$$

When $D \geqslant 3162$, we have

$$
\left|\sum_{d \leqslant D} \frac{\mu(d) \log (D / d)}{d}-1\right| \leqslant \frac{1}{396 \log D} .
$$

Hence, not only do we save a logarithm, but we also save a large constant! Similar results, though less spectacular, are available with the weight $\log ^{2}(D / n)$. They are in principle available for higher powers of $\log (D / n)$, but no one has yet explored this area, nor found some order in it. For identities with $\log (D / n)$, the fractional part that occurred earlier is replaced with the remainder term $\sum_{n \leqslant t} \frac{1}{n}-\log t-\gamma$. We shall see an application of the above theorem in the next section. 
8. Impact on some oscillating multiplicative functions. Musing over the quality of the numerics in Theorem 7.5 the author recently found the following result.

TheOREM 8.1. When $y \geqslant 142130$, we have

$$
\left|\sum_{d>y} \frac{\mu(d)}{d^{2}}\right| \leqslant \frac{0.0195 \log y-0.11}{y(\log y)^{2}} .
$$

When $y \geqslant 59600$, we have

$$
\left|\sum_{d>y} \frac{\mu(d)}{d^{2}}\right| \leqslant \frac{1}{50 y \log y} .
$$

We derive this bound by a simple but unusual process based on the following identity.

Lemma 8.2. Let $(a(d))$ be a complex valued sequence such that $\sum_{d \geqslant 1}|a(d)| / d<\infty$. We have

$$
\sum_{d>y} \frac{a(d)}{d}=\int_{y}^{\infty} \sum_{d \leqslant t} a(d) \log (t / d) \frac{d t}{t^{2}}-\frac{1}{y} \sum_{d \leqslant y} a(d)\left(\log \frac{y}{d}+1\right) .
$$

Proof. We readily find that

$$
\begin{aligned}
\int_{y}^{\infty} \sum_{y<d \leqslant t} a(d) \log (t / d) \frac{d t}{t^{2}} & =\sum_{d>y} a(d) \int_{d}^{\infty} \log (t / d) \frac{d t}{t^{2}} \\
& =\sum_{d>y} \frac{a(d)}{d} \int_{1}^{\infty}(\log t) \frac{d t}{t^{2}}=\sum_{d>y} \frac{a(d)}{d} .
\end{aligned}
$$

Furthermore

$$
\int_{y}^{\infty} \sum_{d \leqslant y} a(d) \log (t / d) \frac{d t}{t^{2}}=\sum_{d \leqslant y} \frac{a(d)}{d} \int_{y / d}^{\infty}(\log t) \frac{d t}{t^{2}}=\sum_{d \leqslant y} \frac{a(d)}{y}\left(\log \frac{y}{d}+1\right) .
$$

On adding both equalities, we get the lemma.

Proof of Theorem 8.1. We first establish the inequality for $y \geqslant 500000$. We employ Lemma 8.2 with $a(d)=\mu(d) / d$ and get

$$
S=\sum_{d>y} \frac{\mu(d)}{d^{2}}=\int_{y}^{\infty} \sum_{d \leqslant t} \frac{\mu(d) \log (t / d)}{d} \frac{d t}{t^{2}}-\sum_{d \leqslant y} \frac{\mu(d)}{d y}\left(\log \frac{y}{d}+1\right) .
$$

Note that the contribution from the main term of $\sum_{d \leqslant t} \mu(d) \log (t / d) / d$ cancels out with the one from $\sum_{d \leqslant y} \mu(d) \log (y / d) / d$ and thus

$$
\begin{aligned}
|S| & \leqslant \int_{y}^{\infty} \frac{(0.00252 \log t-0.0077) d t}{t^{2}(\log t)^{2}}+\frac{0.00252 \log y-0.0077}{y(\log y)^{2}}+\frac{0.0144 \log y-0.1}{y(\log y)^{2}} \\
& \leqslant \int_{y}^{\infty} \frac{(0.00252 \log y-0.0077) d t}{t^{2}(\log t) \log y}+\frac{0.00252 \log y-0.0077}{y(\log y)^{2}}+\frac{0.0144 \log y-0.1}{y(\log y)^{2}} \\
& \leqslant \frac{0.01944 \log y-0.1154}{y(\log y)^{2}} \leqslant \frac{0.0195 \log y-0.11}{y(\log y)^{2}} .
\end{aligned}
$$

We then use a GP-script to extend the result on the interval $\left[142130,10^{9}\right]$, noting that $0.0195 \log y-0.11 \geqslant 0$ when $y \geqslant 282$ and that the function $y \mapsto(0.0195 \log y-$ $0.11) /\left(y(\log y)^{2}\right)$ is first increasing and then decreasing. 
And again, the bound in Theorem 8.1 saves a constant and a logarithm. A careful look at the proof of Theorem 8.1 discloses that the final constant is governed by the summand $\sum_{d \leqslant y} a(d) / y$, meaning that we may benefit from a closer examination of it. This is the meaning of the next result.

ThEOREM 8.3. When $y \geqslant 1043$, we have

$$
\left|\sum_{d>y} \frac{\mu(d)}{d^{2}}-\frac{1}{y} \sum_{d>y} \frac{\mu(d)}{d}\right| \leqslant \frac{0.0051 \log y-0.015}{y(\log y)^{2}} .
$$

When $y \geqslant 222$, we have

$$
\left|\sum_{d>y} \frac{\mu(d)}{d^{2}}-\frac{1}{y} \sum_{d>y} \frac{\mu(d)}{d}\right| \leqslant \frac{1}{196 y \log y} .
$$

Proof. Let us establish the first inequality for $y \geqslant 4000$. We employ Lemma 8.2 with $a(d)=\mu(d) / d$ and get

$$
S=\sum_{d>y} \frac{\mu(d)}{d^{2}}+\frac{1}{y} \sum_{d \leqslant y} \frac{\mu(d)}{d}=\int_{y}^{\infty} \sum_{d \leqslant t} \frac{\mu(d) \log (t / d)}{d} \frac{d t}{t^{2}}-\sum_{d \leqslant y} \frac{\mu(d)}{d y} \log \frac{y}{d} .
$$

Note that $\sum_{d \leqslant y} \mu(d) / d=-\sum_{d>y} \mu(d) / d$. We infer from the above that

$$
\begin{aligned}
|S| & \leqslant \int_{y}^{\infty} \frac{(0.00252 \log t-0.0077) d t}{t^{2}(\log t)^{2}}+\frac{0.00252 \log y-0.0077}{y(\log y)^{2}} \\
& \leqslant \int_{y}^{\infty} \frac{(0.00252 \log y-0.0077) d t}{t^{2}(\log t) \log y}+\frac{0.00252 \log y-0.0077}{y(\log y)^{2}} \\
& \leqslant \frac{0.00504 \log y-0.0154}{y(\log y)^{2}} \leqslant \frac{0.0051 \log y-0.015}{y(\log y)^{2}} .
\end{aligned}
$$

We then use a GP-script to extend the result on the interval $\left[1043,10^{6}\right]$, noting that $0.0051 \log y-0.015 \geqslant 0$ when $y \geqslant 16$ and that the function $y \mapsto(0.0051 \log y-$ $0.015) /\left(y(\log y)^{2}\right)$ is first increasing and then decreasing.

Question 5. Can one relate $\sum_{d>x} \mu(d) / d^{2}$ to $M(y)$ directly via some identity and get a better numerical result than Theorem 8.1?

By reading the proof, it is clear that such identities exist but a clear background like the one we have between $m(x)$ and $M(y)$ is missing.

We are now ready to prove Theorem 3.2

\section{Impact on some non-negative multiplicative functions}

Proof of Theorem 3.2. We start with an easy observation.

LEMma 9.1. With $R(X)=\sum_{n \leqslant X} 1 / n-\log X-\gamma$, we have

$$
\left|\log \frac{A_{0}}{\left[A_{0}\right]}-R\left(\left[A_{0}\right]\right)\right| \leqslant \frac{1 / 2}{\left[A_{0}\right]} .
$$

When $\left[A_{0}\right]=1\left(\right.$ resp. $\left[A_{0}\right]=2$, resp. $\left.\left[A_{0}\right]=3\right)$, we can replace $1 / 2$ by 0.3 (resp. 0.36 , resp. 0.4). 
Proof. We recall that, with $N=\left[A_{0}\right]$, we have

$$
R(N)=\frac{1}{2 N}-\frac{1}{12 N^{2}}+\mathcal{O}^{*}\left(\frac{1}{60 N^{4}}\right)
$$

and thus

$$
\log \frac{A_{0}}{N}-R(N)=\log \frac{A_{0}}{N}-\frac{1}{2 N}+\frac{1}{12 N^{2}}+\mathcal{O}^{*}\left(\frac{1}{60 N^{4}}\right)
$$

from which we easily deduce that

$$
\left|\log \frac{A_{0}}{N}-R(N)\right| \leqslant \frac{\left|N \log \left(1+N^{-1}\right)-\frac{1}{2}+\frac{1}{12 N}\right|+\frac{1}{60 N^{3}}}{N} .
$$

Corollary 1.2 only proves our result for $D \in\left[82005,5 \cdot 10^{7}\right]$. So we have recourse to direct computations.

LEMma 9.2. For $D \leqslant 10^{11}$, we have

$$
\sum_{d \leqslant D} \frac{\mu^{2}(d)}{d}=\frac{6}{\pi^{2}}\left(\log D+C_{2}\right)+\mathcal{O}^{*}\left(\frac{1}{D^{3 / 4}}\right)
$$

The constant in the numerator of $1 / D^{3 / 4}$ oscillates in this range between $0.074 \cdots$ (around $D=7.214 \cdot 10^{10} \pm 10^{7}$ ) and $0.977 \cdots$ (around $D=3.63 \cdot 10^{9} \pm 10^{7}$ ). It still seems to decrease slowly. We adapted the GP/Pari script described in the proof of Lemma 2.1 of [34] and let it run for some days (on a desktop computer having only 8 Gigabytes of RAM, the computation was split to intervals of length $2 \cdot 10^{7}$ ).

This lemma proves Theorem 3.2 for $D \in\left[5000,10^{11}\right]$. When $D \geqslant 10^{11}$, we use our Unbalanced Dirichlet Hyperbola formula (Theorem 4.1) with the multiplicative function $g$ being defined by

$$
g\left(p^{2}\right)=-1, \quad \forall k \in\{1\} \cup\left(\left[3, \infty[\cap \mathbb{N}), \quad g\left(p^{k}\right)=0 .\right.\right.
$$

Hence we need to evaluate

$$
G^{\sharp}(x)=\sum_{m^{2}>x} \frac{\mu(m)}{m^{2}}
$$

for which Theorem 8.1 gives $G^{\sharp}(x)=\mathcal{O}^{*}(1 /(25 \sqrt{x} \log x))$ when $x \geqslant(59600)^{2}$. Whence we find that (in the notation of Theorem 4.1), provided that $\sqrt{D / A_{0}} \geqslant 1700$ :

$$
\begin{aligned}
\Re \sqrt{D \log D} \leqslant & 0.04 \sum_{1 \leqslant a \leqslant A_{0}} \frac{\sqrt{a} \sqrt{\log D}}{a \log (D / a)}+\frac{0.04 \sqrt{A_{0} \log D}}{\log \left(D / A_{0}\right)}\left|\log \frac{A_{0}}{\left[A_{0}\right]}-R\left(\left[A_{0}\right]\right)\right| \\
& +\frac{0.504 \times 0.62 \sqrt{\log D}}{\sqrt{A_{0}}}+0.04 \sqrt{D \log D} \int_{D / A_{0}}^{\infty} d t /\left(t^{3 / 2} \log t\right) .
\end{aligned}
$$

The quantity $\int_{D / A_{0}}^{\infty} d t /\left[t^{3 / 2} \log t\right]$ is dealt with via

$$
\int_{D / A_{0}}^{\infty} d t /\left[t^{3 / 2} \log t\right]=\int_{\frac{1}{2} \log \left(D / A_{0}\right)} e^{-u} \frac{d u}{u} \leqslant \frac{2}{\sqrt{D} \log \left(D / A_{0}\right)}\left(1-\frac{2}{\log \left(D / A_{0}\right)}+\frac{8}{\log \left(D / A_{0}\right)}\right) .
$$


Let $A_{1}$ be an integer parameter (we shall select $A_{1}=71$ ). We single out the term with $a \leqslant A_{1}$ and use the inequality $\sum_{A_{1}+1 \leqslant a \leqslant A_{0}} 1 / \sqrt{a} \leqslant \int_{A_{1}}^{A_{0}} d t / \sqrt{t} \leqslant \frac{1}{2}\left(\sqrt{A_{0}}-\sqrt{A_{1}}\right)$. This yields

$$
\begin{aligned}
& \Re \sqrt{D} \log D \\
& \leqslant \sum_{1 \leqslant a \leqslant A_{1}} \frac{0.04}{\sqrt{\log (D / a)}}+\frac{0.04}{2} \frac{\sqrt{A_{0}}-\sqrt{A_{1}}}{\sqrt{\log D}-\frac{\log A_{0}}{\sqrt{\log D}}+\frac{0.04 \sqrt{A_{0}}}{\sqrt{\log D}-\frac{\log A_{0}}{\sqrt{\log D}}} \frac{1 / 2}{A_{0}-1}} \\
& \quad+\frac{0.504 \times 0.62 \sqrt{\log D}}{\sqrt{A_{0}}}+0.08 \frac{\sqrt{A_{0} \log D}}{\log \left(D / A_{0}\right)}\left(1-\frac{2}{\log \left(D / A_{0}\right)}+\frac{8}{\log \left(D / A_{0}\right)}\right) .
\end{aligned}
$$

We choose $A_{0}=0.504 \frac{0.62}{0.04+\frac{0.04}{2}} \log D \geqslant 71$ when $D \geqslant \exp (23)$, obtaining the final constant 0.347 , which we majorize by $7 / 20$. This 0.347 would only be replaced by 0.345 if we were assuming that $D \geqslant 10^{11}$. A numerical verification using GP/Pari [27] enables us to finish the proof.

The proof of Corollary 3.3 follows from the above result when $D \geqslant 10^{10}$. Lemma 9.2 proves it for $D \in\left[36000,10^{11}\right]$ and a finite verification concludes.

Proof of Theorem 3.2-continuation. Let us now turn to the summatory function of $\mu^{2}(d) / \varphi(d)$. There are two ways to handle the situation:

- Compare the summand to $1 / d$.

- Compare the summand to $\mu^{2}(d) / d$.

The first path consists in using our Unbalanced Dirichlet Hyperbola formula (Theorem 4.1. This is the one we followed with P. Akhilesh in 38] and we show here how the proof simplifies when we use the second path.

We start with a finite verification.

LEMmA 9.3. When $D \in\left[1,2 \cdot 10^{9}\right]$, we have

$$
\sum_{d \leqslant D} \frac{\mu^{2}(d)}{\varphi(d)}=\log D+c_{0}+\mathcal{O}^{*}\left(2.2 / D^{3 / 4}\right) .
$$

The constant 2.2 above is an upper bound for a function that oscillates, when $D$ ranges $\left[1,10^{9}\right]$, between 0.52 and 2.16 , the maximum being away from the beginning (after $10^{6}$ ).

We relate $\mu^{2}(d) / \varphi(d)$ to $\mu^{2}(d) / d$ in the following, next-to-trivial lemma.

LEMMA 9.4. For any integer $n \geqslant 1$, we have

$$
\mu^{2}(n) \frac{n}{\varphi(n)}=\sum_{\ell m=k} \theta(\ell) \mu^{2}(m)
$$

where $\theta$ is the multiplicative function defined on the prime powers $p^{k}$ by

$$
\theta\left(p^{k}\right)=(-1)^{k+1} /(p-1)
$$

Proof. Both functions being multiplicative, it is enough to check the identity on the $p$-components of the Dirichlet series. To do so, it is enough to note that

$$
\frac{1+\frac{p}{p-1} X}{1+X}=1+\frac{1}{p-1} \frac{X}{1+X}=1+\sum_{k \geqslant 1} \frac{(-1)^{k+1} X^{k}}{p-1} \text {. }
$$


As it turns out, the function $\theta$ that appears is much more difficult to handle precisely than (the author) expected.

On a troublesome special function. On denoting by $k(n)$ the squarefree kernel of $n$ (sometimes called the core of $n$ ), i.e. $k(n)=\prod_{p \mid n} p$, we find that

$$
\theta(n)=\frac{\lambda(n k(n))}{\varphi(k(n))} .
$$

The simplest function $1 / k(n)$ has already been the subject of numerous questions; its average order is known (see [1] and [40]) but difficult to get. We have

$$
\sum_{n \leqslant N} 1 / k(n)=\exp ((1+o(1)) \sqrt{8(\log N) / \log \log N)}
$$

An inspection of the proof of [11] discloses that a similar asymptotic is true for the average $\sum_{n \leqslant N} 1 / \varphi(k(n))$. In particular, it is not of the shape $C \log N$, which means the convolution method (which would compare it to a simpler function, say $1 / n$ ) will not work here. The function $\theta$, being not especially non-negative, is not covered by these results.

Since we do not know how to be precise with the function $\theta$, we will employ Rankin's trick. Notice that both Dirichlet series

$$
\left\{\begin{array}{l}
T(s)=\sum_{n \geqslant 1} \frac{\theta(n)}{n^{s}}=\prod_{p \geqslant 2}\left(1+\frac{1}{(p-1)\left(p^{s}+1\right)}\right), \\
T^{*}(s)=\sum_{n \geqslant 1} \frac{|\theta(n)|}{n^{s}}=\prod_{p \geqslant 2}\left(1+\frac{1}{(p-1)\left(p^{s}-1\right)}\right)
\end{array}\right.
$$

are absolutely convergent for $\Re s>0$ with $T(1)=\pi^{2} / 6$. We also readily compute that

$$
\max _{\Re s \geqslant 1 / 2}\left|T^{*}(s)\right|=\prod_{p \geqslant 2}\left(1+\frac{1}{(p-1)\left(p^{1 / 2}-1\right)}\right) \leqslant 10 .
$$

Question 6. Can one find an efficient bound for $\left|\sum_{\ell>t} \theta(\ell) / \ell\right|$ ?

We will only use the upper bound $\sum_{\ell>t}|\theta(\ell)| / \ell$ for $\left|\sum_{\ell>t} \theta(\ell) / \ell\right|$ and then use Rankin's trick, losing at least the sign of $\theta(\ell)$ in the process.

Resuming the proof. As a consequence of Lemma 9.4, we find that

$$
\sum_{d \leqslant D} \frac{\mu^{2}(d)}{\varphi(d)}=\sum_{\ell \geqslant 1} \frac{\theta(\ell)}{\ell} \sum_{m \leqslant D / \ell} \frac{\mu^{2}(m)}{m} .
$$

We use our Generalized Unbalanced Hyperbola Formula (Theorem 4.2 and get

$$
\begin{aligned}
\sum_{d \leqslant D} \frac{\mu^{2}(d)}{\varphi(d)}= & \sum_{\ell \geqslant 1} \frac{\theta(\ell)}{\ell} \frac{6}{\pi^{2}}\left(\log \frac{D}{\ell}+C_{2}\right)+\frac{6}{\pi^{2}} \int_{D / A_{0}}^{\infty} G^{\sharp}(t) \frac{d t}{t} \\
& +\mathcal{O}^{*}\left(\left|\sum_{a \leqslant A_{0}} \frac{\mu^{2}(a)}{a} G^{\sharp}\left(\frac{D}{a}\right)+G^{\sharp}\left(\frac{D}{A_{0}}\right)\left(\frac{6}{\pi^{2}} \log \frac{A_{0}}{\left[A_{0}\right]}-R_{\mu^{2}}\left(\left[A_{0}\right]\right)\right)\right|\right) \\
& +\mathcal{O}^{*}\left(\sum_{m \leqslant D / A_{0}}\left|\frac{\theta(m)}{m} R_{h}\left(\frac{D}{m}\right)\right|\right)
\end{aligned}
$$


where now we have set

$$
G^{\sharp}(x)=\sum_{m>x} \theta(m) / m .
$$

Notice that, for any $\alpha_{0} \in[0,1)$, we have $\left|G^{\sharp}(x)\right| \leqslant T^{*}\left(1-\alpha_{0}\right) / x^{\alpha_{0}}$. By Lemma 9.3 , we can assume that $D \geqslant 10^{9}$. We get

$$
\begin{aligned}
\sum_{d \leqslant D} & \frac{\mu^{2}(d)}{\varphi(d)} \\
= & \log D+c_{0}+\mathcal{O}^{*}\left(\frac{6}{\pi^{2}} \frac{T^{*}\left(1-\alpha_{0}\right) A_{0}^{\alpha_{0}}}{\alpha_{0} D^{\alpha_{0}}}+\sum_{m \leqslant D / A_{0}}\left|\frac{\theta(m)}{m} R_{h}\left(\frac{D}{m}\right)\right|\right) \\
& +\mathcal{O}^{*}\left(\frac{T^{*}\left(1-\alpha_{0}\right)}{D^{\alpha_{0}}} \sum_{a \leqslant A_{0}-1} \frac{\mu^{2}(a)}{a^{1-\alpha_{0}}}+\frac{T^{*}\left(1-\alpha_{0}\right)}{\left(D / A_{0}\right)^{\alpha_{0}}}\left|1+\frac{6}{\pi^{2}} \log \frac{A_{0}}{\left[A_{0}\right]}-R_{\mu^{2}}\left(\left[A_{0}\right]\right)\right|\right) .
\end{aligned}
$$

We select $A_{0}=1$ and notice that $R_{\mu^{2}}(1)=1-\frac{6}{\pi^{2}} C_{2}$. Let us take a parameter $L=D^{\beta}$ for some $\beta \in(0,1)$. We note that when $D / M_{0} \geqslant 1665$, we have

$$
\begin{aligned}
& \sqrt{D} \sum_{m \leqslant D}\left|\frac{\theta(m)}{m} R_{h}\left(\frac{D}{m}\right)\right| \leqslant \frac{7}{20} \sum_{m \leqslant M_{0}} \frac{|\theta(m)|}{\sqrt{m \log (D / m)}} \\
+ & \left(T^{*}(1 / 2)-\sum_{m \leqslant M_{0}} \frac{|\theta(m)|}{\sqrt{m}}\right) \frac{0.56}{\sqrt{\log (D / L)}}+0.43\left(T^{*}\left(\frac{1}{2}-\alpha\right)-\sum_{m \leqslant M_{0}} \frac{|\theta(m)|}{m^{1 / 2-\alpha}}\right) \frac{1}{\sqrt{D} L^{\alpha}}
\end{aligned}
$$

by using Theorem 3.2 when $m \in\left[1, M_{0}\right]$ with the constant $7 / 20$, then Theorem 3.2 when $m \in\left[M_{0}+1, L\right]$ this time with the constant 0.56 , and then the last part of Corollary 1.2 when $m \in(L, D]$. With $D \geqslant 10^{9}$, we select $\alpha_{0}=0.81, M_{0}=80, \alpha=0.26, \beta=0.78$ and get a constant $\leqslant 11$.

Concerning the error term in $1 / \sqrt{D}$, the preceding result proves it when $D \geqslant 10^{9}$. Lemma 9.3 enables us to extend it to $D \geqslant 3$ and a direct verification concludes.

10. Proof of Corollary 3.4. We start with an easy lemma.

Lemma 10.1. When $D \geqslant 1665$, we have

$$
\int_{1665}^{D} \frac{d t}{\sqrt{t \log t}} \leqslant \frac{2 \sqrt{D}(\log D-2)}{\sqrt{\log D}(\log D-3)}-29 .
$$

Proof. We first note that

$$
\int_{1665}^{D} \frac{d t}{\sqrt{t \log ^{3} t}} \leqslant \frac{2 \sqrt{D}}{(\log D)^{3 / 2}}+\int_{1665}^{D} \frac{3 d t}{\sqrt{t \log ^{5} t}} \leqslant \frac{2 \sqrt{D}}{(\log D)^{3 / 2}}+\frac{3}{\log D} \int_{1665}^{D} \frac{3 d t}{\sqrt{t \log ^{3} t}}
$$

whence

$$
\int_{1665}^{D} \frac{d t}{\sqrt{t \log ^{3} t}} \leqslant \frac{2 \sqrt{D}}{\sqrt{\log D}(\log D-3)} .
$$

Now, an integration by parts again gives us

$$
\int_{1665}^{D} \frac{d t}{\sqrt{t \log t}}=\frac{2 \sqrt{D}}{\sqrt{\log D}}-\frac{2 \sqrt{1665}}{\sqrt{\log 1665}}+\int_{1665}^{D} \frac{d t}{\sqrt{t \log ^{3} t}}
$$

and the lemma follows readily. 
Let us now prove Corollary 3.4 . We find that

$$
\begin{aligned}
\sum_{d \leqslant D} \mu^{2}(d)= & D \sum_{d \leqslant D} \frac{\mu^{2}(d)}{d}-\int_{0}^{D} \sum_{d \leqslant t} \frac{\mu^{2}(d)}{d} d t \\
= & D\left(\sum_{d \leqslant D} \frac{\mu^{2}(d)}{d}-\frac{6}{\pi^{2}}\left(\log D+C_{2}\right)\right)-\frac{6}{\pi^{2}}\left(D \log D-D+C_{2} D\right) \\
& -\int_{0}^{D}\left(\sum_{d \leqslant t} \frac{\mu^{2}(d)}{d}-\frac{6}{\pi^{2}}\left(\log t+C_{2}\right)\right) d t \\
= & \frac{6}{\pi^{2}} D+\mathcal{O}^{*}\left(\frac{7 \sqrt{D} / 20}{\sqrt{\log D}}\right)-\int_{0}^{1665}\left(\sum_{d \leqslant t} \frac{\mu^{2}(d)}{d}-\frac{6}{\pi^{2}}\left(\log t+C_{2}\right)\right) d t \\
& +\mathcal{O}^{*}\left(\int_{1665}^{D} \frac{7 / 20}{\sqrt{t \log t}} d t\right) .
\end{aligned}
$$

We directly compute that

$$
\begin{aligned}
& \int_{0}^{1665}\left(\sum_{d \leqslant t} \frac{\mu^{2}(d)}{d}-\frac{6}{\pi^{2}}\left(\log t+C_{2}\right)\right) d t \\
& \quad=\sum_{d \leqslant 1665} \frac{\mu^{2}(d)(1665-d)}{d}-\frac{6}{\pi^{2}}\left(1665 \log (1665)-1665+1665 C_{2}\right)=-0.7248 \cdots .
\end{aligned}
$$

We also appeal to the above lemma and deduce that

$$
\sum_{d \leqslant D} \mu^{2}(d)=\frac{6}{\pi^{2}} D+\mathcal{O}^{*}\left(\frac{\frac{7}{20} \sqrt{D}(3 \log D-7)}{\sqrt{\log D}(\log D-3)}\right) .
$$

We can assume that $D \geqslant 10^{32}$ and get that this error term is also $\mathcal{O}^{*}(1.06 \sqrt{D} / \sqrt{\log D})$. When $82005 \leqslant D \leqslant 10^{32}$, our estimate is a consequence of Theorem 1.1 and a readilyachieved finite verification justifies the extension of the range to $D>1$.

11. The Covering Remainder Lemma. We work under the assumption detailed just before the statement of Theorem 4.2

Lemma 11.1 (The Covering Remainder Lemma). Let $(g(m))_{m \geqslant 1}$ be a sequence of complex numbers such that $\sum_{m \geqslant 1} g(m) / m$ converges. Define $G^{\sharp}(x)=\sum_{m>x} g(m) / m$. Let $A_{0} \geqslant 1$ be a real number and let $\left[A_{0}\right]$ denote its integer part. We have

$$
\begin{aligned}
\sum_{X / A_{0}<m \leqslant X} \frac{g(m)}{m} R_{h}\left(\frac{X}{m}\right)= & K_{0} K_{1} G^{\sharp}(X)+K_{0} \int_{1}^{A_{0}} G^{\sharp}\left(\frac{X}{t}\right) \frac{d t}{t}-\sum_{1 \leqslant a \leqslant A_{0}} \frac{h(a)}{a} G^{\sharp}\left(\frac{X}{a}\right) \\
& -G^{\sharp}\left(\frac{X}{A_{0}}\right)\left(K_{0} \log \frac{A_{0}}{\left[A_{0}\right]}-R_{h}\left(\left[A_{0}\right]\right)\right) .
\end{aligned}
$$

Proof. We set $B=\left[A_{0}\right]$ to ease the typing. When $a$ is a positive integer, $b \in[a, a+1]$, and $m$ is inside $(X / b, X / a]$, we have

$$
K_{0}\left(\log \frac{X}{m}+K_{1}\right)+R_{h}(X / m)=\sum_{n \leqslant X / m} \frac{h(n)}{n}=\sum_{n \leqslant a} \frac{h(n)}{n}=K_{0}\left(\log a+K_{1}\right)+R_{h}(a)
$$


from which we infer that $R_{h}(X / m)=R_{h}(a)-K_{0} \int_{a}^{X / m} d t / t$. This implies that

$$
\sum_{X / b<m \leqslant X / a} \frac{g(m)}{m} R_{h}(X / m)=\sum_{X / b<m \leqslant X / a} \frac{g(m)}{m} R_{h}(a)-K_{0} \int_{a}^{b} \sum_{X / b<m \leqslant X / t} \frac{g(m)}{m} \frac{d t}{t} .
$$

We see at this level that the proof of the Covering Remainder Lemma of [38] applies, simply multiplying the integral by $K_{0}$, replacing $R$ by $R_{h}$. Since this part is not long, we copy it for the comfort of the reader.

We sum the construction step over $a \in\{1, \ldots, B\}$ with the choice $b=\min \left(a+1, A_{0}\right)$. On using the notation $G^{\sharp}$, we get

$$
\begin{aligned}
\sum_{X / A_{0}<m \leqslant X} \frac{g(m)}{m} & R_{h}(X / m) \\
= & \sum_{a \leqslant B}\left(G^{\sharp}\left(\frac{X}{\min \left(a+1, A_{0}\right)}\right)-G^{\sharp}\left(\frac{X}{a}\right)\right) R_{h}(a) \\
& +K_{0} \sum_{a \leqslant B} \int_{a}^{\min \left(a+1, A_{0}\right)}\left(G^{\sharp}\left(\frac{X}{t}\right)-G^{\sharp}\left(\frac{X}{\min \left(a+1, A_{0}\right)}\right)\right) \frac{d t}{t} .
\end{aligned}
$$

Some shuffling is called for. Here is the first step:

$$
\begin{aligned}
\sum_{X / A_{0}<m \leqslant X} & \frac{g(m)}{m} R_{h}(X / m) \\
= & \sum_{2 \leqslant a \leqslant B+1} G^{\sharp}\left(\frac{X}{\min \left(a, A_{0}\right)}\right) R_{h}(a-1)-\sum_{a \leqslant B} G^{\sharp}\left(\frac{X}{a}\right) R_{h}(a) \\
& +K_{0} \int_{1}^{A_{0}} G^{\sharp}\left(\frac{X}{t}\right) \frac{d t}{t}-K_{0} \sum_{a \leqslant B} G^{\sharp}\left(\frac{X}{\min \left(A_{0}, a+1\right)}\right) \log \frac{\min \left(A_{0}, a+1\right)}{a}
\end{aligned}
$$

which we rewrite in the form (we set $R(0)=0$ )

$$
\begin{aligned}
\sum_{X / A_{0}<m \leqslant X} & \frac{g(m)}{m} R_{h}(X / m) \\
= & \sum_{1 \leqslant a \leqslant B} G^{\sharp}\left(\frac{X}{a}\right)\left(R_{h}(a-1)-R_{h}(a)\right)+G^{\sharp}\left(\frac{X}{A_{0}}\right) R_{h}(B) \\
& +K_{0} \int_{1}^{A_{0}} G^{\sharp}\left(\frac{X}{t}\right) \frac{d t}{t}-K_{0} \sum_{a \leqslant B} G^{\sharp}\left(\frac{X}{\min \left(A_{0}, a+1\right)}\right) \log \frac{\min \left(A_{0}, a+1\right)}{a} .
\end{aligned}
$$

Here is the second step:

$$
\begin{aligned}
\sum_{X / A_{0}<m \leqslant X} \frac{g(m)}{m} R_{h}(X / m)= & -G^{\sharp}(X) R_{h}(1)+K_{0} \int_{1}^{A_{0}} G^{\sharp}\left(\frac{X}{t}\right) \frac{d t}{t} \\
& +\sum_{2 \leqslant a \leqslant B} G^{\sharp}\left(\frac{X}{a}\right)\left(R_{h}(a-1)-R_{h}(a)-K_{0} \log \frac{a}{a-1}\right) \\
& +G^{\sharp}\left(\frac{X}{A_{0}}\right)\left(R_{h}(B)-K_{0} \log \frac{A_{0}}{B}\right) .
\end{aligned}
$$


It is then obvious to establish the claimed formula since $R_{h}(1)=h(1)-K_{0} K_{1}$ and $-h(a) / a=R(a-1)+K_{0} \log (a-1)-\left(R(a)+K_{0} \log (a)\right)$ when $a \geqslant 2$.

12. Proof of Corollary 1.2. We simply use the following identity which comes from an integration by parts:

$$
\sum_{d \leqslant D} \frac{\mu^{2}(d)}{d}=\frac{6}{\pi^{2}}\left(\log D+C_{2}\right)+\frac{\sum_{d \leqslant D} \mu^{2}(d)-\frac{6}{\pi^{2}} D}{D}-\int_{D}^{\infty}\left(\sum_{d \leqslant t} \mu^{2}(d)-\frac{6}{\pi^{2}} t\right) \frac{d t}{t^{2}} .
$$

On plugging the estimate given by Theorem 1.1 inside, we get our result. The constant term that appears is simply identified.

The constant in the numerator of $0.43 / \sqrt{D}$ can be reduced by this process to

$$
\left(\frac{6}{\pi^{2}}\left(\log 5+C_{2}\right)-\frac{11}{6}\right) \sqrt{5}=0.422 \cdots
$$

but no more.

Acknowledgments. Thanks are due to the anonymous referee for having read this work very carefully and having proposed useful modifications.

This work has been partly supported by the Indo-French Centre for the Promotion of Advanced Research - CEFIPRA, project No 5401-1.

\section{References}

[1] A. Axer, Beitrag zur Kenntnis der zahlentheoretischen Funktionen $\mu(n)$ und $\lambda(n)$, Prace Matematyczno-Fizyczne (1910), 65-95.

[2] L. Báez-Duarte, M. Balazard, B. Landreau, É. Saias, Notes sur la fonction $\zeta$ de Riemann. III, Adv. Math. 149 (2000), 130-144.

[3] M. Balazard, Remarques élémentaires sur la fonction de Moebius, private communication, 2011, hal-00732694.

[4] M. Balazard, Elementary remarks on Möbius' function, Tr. Mat. Inst. Steklova 276 (2012), 39-45; English transl.: Proc. Steklov Inst. Math. 276 (2012), 33-39.

[5] O. Bordellès, An explicit Mertens' type inequality for arithmetic progressions, JIPAM. J. Inequal. Pure Appl. Math. 6 (2005), Article 67.

[6] J. Büthe, A Brun-Titchmarsh inequality for weighted sums over prime numbers, Acta Arith. 166 (2014), 289-299.

[7] M. Cipu, Further remarks on Diophantine quintuples, Acta Arith. 168 (2015), 201-219.

[8] H. Cohen, F. Dress, Estimations numériques du reste de la fonction sommatoire relative aux entiers sans facteur carré, in: Colloque de théorie analytique des nombres (Marseille, 1985), Publ. Math. Orsay 88-02, Univ. Paris XI Orsay, 1988, 73-76.

[9] H. Cohen, F. Dress, M. El Marraki, Explicit estimates for summatory functions linked to the Möbius $\mu$-function, Funct. Approx. Comment. Math. 37 (2007), 51-63.

[10] F. Daval, Private communication, 2017.

[11] N. G. de Bruijn, On the number of integers $\leqslant x$ whose prime factors divide $n$, Illinois J. Math. 6 (1962), 137-141.

[12] H. G. Diamond, W.-B. Zhang, A PNT equivalence for Beurling numbers, Funct. Approx. Comment. Math. 46 (2012), 225-234. 
[13] F. Dress, M. El Marraki, Fonction sommatoire de la fonction de Möbius II. Majorations asymptotiques élémentaires, Experiment. Math. 2 (1993), 99-112.

[14] A. W. Dudek, On the sum of a prime and a square-free number, Ramanujan J. 42 (2017), 233-240.

[15] A. W. Dudek, D. J. Platt, On the sum of the square of a prime and a square-free number, LMS J. Comput. Math. 19 (2016), 16-24.

[16] P. Dusart, Explicit estimates of some functions over primes, Ramanujan J. 45 (2018), 227-251.

[17] M. El Marraki, Fonction sommatoire de la fonction $\mu$ de Möbius III. Majorations asymptotiques effectives fortes, J. Théor. Nombres Bordeaux 7 (1995), 407-433.

[18] M. El Marraki, Majorations de la fonction sommatoire de la fonction $\frac{\mu(n)}{n}$, Univ. Bordeaux 1, Pré-publication(96-8), 1996.

[19] L. Faber, H. Kadiri, New bounds for $\psi(x)$, Math. Comp. 84 (2015), 1339-1357.

[20] I. C. Gohberg, M. G. Kreĭn, Introduction to the theory of linear nonselfadjoint operators, Transl. Math. Monogr. 18, Amer. Math. Soc., Providence, R.I., 1969.

[21] X. Gourdon, The $10^{13}$ first zeros of the Riemann Zeta Function and zeros computations at very large height, 2004, http://numbers.computation.free.fr/Constants/Miscellaneous/zetazeros1e13-1e24.pdf

[22] A. Kienast, Über die Äquivalenz zweier Ergebnisse der analytischen Zahlentheorie, Math. Ann. 95 (1926), 427-445.

[23] E. Landau, Neuer Beweis der Gleichung $\sum_{k=1}^{\infty} \frac{\mu(k)}{k}=0$, Berlin, 1899, 16 S. gr. $8^{\circ}$.

[24] E. Landau, Über einige neuere Grenzwertsätze, Rend. Circ. Mat. Palermo 34 (1912), 121131.

[25] R. A. MacLeod, A curious identity for the Möbius function, Utilitas Math. 46 (1994), 91-95.

[26] H. L. Montgomery, Fluctuations in the mean of Euler's phi function, Proc. Indian Acad. Sci. Math. Sci. 97 (1987), 239-245.

[27] The PARI Group, PARI/GP version 2.7.0, Bordeaux, 2014, pari.math.u-bordeaux.fr/

[28] D. J. Platt, Numerical computations concerning the GRH, Ph.D. thesis, 2013, http://arxiv.org/abs/1305.3087.

[29] D. J. Platt, Isolating some non-trivial zeros of zeta, Math. Comp. 86 (2017), 2449-2467.

[30] D. J. Platt, O. Ramaré, Explicit estimates: from $\Lambda(n)$ in arithmetic progressions to $\Lambda(n) / n$, Exp. Math. 26 (2017), 77-92.

[31] O. Ramaré, On Šnirel'man's constant, Ann. Scuola Norm. Sup. Pisa Cl. Sci. (4) 21 (1995), 645-706,

[32] O. Ramaré, Explicit estimates for the summatory function of $\Lambda(n) / n$ from the one of $\Lambda(n)$, Acta Arith. 159 (2013), 113-122.

[33] O. Ramaré, From explicit estimates for the primes to explicit estimates for the Möbius function, Acta Arith. 157 (2013), 365-379.

[34] O. Ramaré, Explicit estimates on the summatory functions of the Möbius function with coprimality restrictions, Acta Arith. 165 (2014), 1-10.

[35] O. Ramaré, Explicit estimates on several summatory functions involving the Moebius function, Math. Comp. 84 (2015), 1359-1387.

[36] O. Ramaré, An explicit density estimate for Dirichlet L-series, Math. Comp. 85 (2016), 325-356.

[37] O. Ramaré, On the missing log factor, in: Ergodic Theory and Dynamical Systems in their Interactions with Arithmetics and Combinatorics, Lecture Notes in Math. 2213, Springer, Cham, 2018, 293-315. 
[38] O. Ramaré, P. Akhilesh, Explicit averages of non-negative multiplicative functions: going beyond the main term, Colloq. Math. 147 (2017), 275-313.

[39] H. Riesel, R. C. Vaughan, On sums of primes, Ark. Mat. 21 (1983), 46-74.

[40] O. Robert, G. Tenenbaum, Sur la répartition du noyau d'un entier, Indag. Math. (N.S.) 24 (2013), 802-914.

[41] J. B. Rosser, Explicit bounds for some functions of prime numbers, Amer. J. Math. 63 (1941), 211-232.

[42] J. B. Rosser, L. Schoenfeld, Approximate formulas for some functions of prime numbers, Illinois J. Math. 6 (1962), 64-94.

[43] J. B. Rosser, L. Schoenfeld, Sharper bounds for the Chebyshev functions $\vartheta(x)$ and $\psi(x)$, Math. Comp. 29 (1975), 243-269.

[44] L. Schoenfeld, An improved estimate for the summatory function of the Möbius function, Acta Arith. 15 (1969), 221-233.

[45] D. W. Swann, Some new classes of kernels whose Fredholm determinants have order less than one, Trans. Amer. Math. Soc. 160 (1971), 427-435.

[46] E. Treviño, The least k-th power non-residue, J. Number Theory 149 (2015), 201-224.

[47] T. Trudgian, Bounds on the number of Diophantine quintuples, J. Number Theory 157 (2015), 233-249.

[48] T. Trudgian, Explicit bounds on the logarithmic derivative and the reciprocal of the Riemann zeta-function, Funct. Approx. Comment. Math. 52 (2015), 253-261.

[49] R. Vanlalnagaia, Explicit estimates for some summatory functions of primes, Integers 17 (2017), no. A11.

[50] W.-B. Zhang, A generalization of Halász's theorem to Beurling's generalized integers and its application, Illinois J. Math. 31 (1987), 645-664. 